\title{
O ESTUDO DA DIFERENÇA DE POTENCIAL (DDP) A PARTIR DE REAÇÃO DE OXIRREDUÇÃO (PILHA) E APLICAÇÃO DA MODELAGEM E SIMULAÇÃO COMPUTACIONAL
}

\author{
Yan Pedro Ulrich Mendes Ferreira \\ Graduando em engenharia química no Instituto de \\ Química da Universidade do Estado do Rio de Janeiro \\ $\triangle$ yanulrich.uerj@gmail.com \\ Alan Freitas Machado \\ Professor do Departamento de Física Teórica do Instituto de \\ Física da Universidade do Estado do Rio de Janeiro \\ 凶alanfmac@gmail.com \\ Diego Barbosa Moura \\ Professor Assistente do Colégio Universitário \\ Geraldo Reis da Universidade Federal Fluminense

\section{Claudio Elias da Silva} \\ Professor Associado do Departamento de Eletrônica Quântica do \\ Instituto de Física da Universidade do Estado do Rio de Janeiro
}

\section{Resumo:}

A proposta desse trabalho é descrever uma experiência bem sucedida de ensino e aprendizagem aplicada a um grupo de estudantes do ensino médio com emprego do software educacional Modellus para simular uma reação de oxirredução em meio de ácido cítrico com eletrodos de ferro e cobre (pilha) e com isto objetiva-se estudar o comportamento do sistema através dos valores da energia potencial (DDP) que são fornecidos pela modelagem em função do volume de solução e confrontá-los com os valores obtidos experimentalmente. Observa-se então que o processo de criação da modelagem computacional vai além de uma melhora apenas nos conhecimentos teóricos dos alunos, estimulando-os a questionarem sobre toda a teoria envolvida no processo de criação da modelagem, propondo sugestões e buscando soluções para que contornem alguns problemas e limitações do software. Sendo assim, os alunos são levados para além de uma aprendizagem puramente teórica e abstrata, possibilitando que se apropriem dos fundamentos matemáticos e físicos envolvidos no processo de ensino e aprendizagem, desenvolvendo um olhar crítico e estabelecendo sua autonomia através do diálogo entre seus pares e o professor.

Palavras-chave: Software educacional; Aprendizagem; Modelagem computacional; Modellus.

\section{STUDY OF THE POTENTIAL DIFFERENCE (DDP) GENERATED BY AN REACTION OXIDATION-REDUCTION (CEEL) WITH THE USE OF COMPUTATIONAL MODELING}

\begin{abstract}
:
With the rising and intensification of production's activities, as industries, the deforestation taxes show to be on an alarming level. It is wide spread known the consequences that
\end{abstract}


deforestation might cause in metropolitan areas. In order to evalue the consequences of this growing phenomenon, is possible to use a role of ambiental impacts evaluations techniques. One of them is the Preliminary Analysis of Danger (PAD), which is based on qualitative and statistics analysis and might be used in association with other ambiental impacts evaluations techniques. It's goal is to analyze dangers in potential, it's causes and consequences, in which is also done an associated risk analysis, which the last is the association between the classifications of frequency and severity, and, in the end, it gives suggestions of measures to avoid these dangers (undesired events). In this study, six dangers have been identified, in which none of them present despicable or low risk (0\%), $16 \%$ present medium or high risk and $66 \%$ present critic risk. These datas point out that the deforestation situation might cause dangers with great consequences to Petropolis' metropolitan area, just as floodings and earth slidings, which higthlights the urgency of management of the area.

Keywords: Educational software; learning; Computational modeling; Modellus.

\title{
ESTUDIO DE LA DIFERENCIA DE POTENCIAL (DDP) GENERADO POR UNA REACCIÓN REDOX (BATERÍA) UTILIZANDO EL MODELADO COMPUTACIONAL
}

\begin{abstract}
Resumen:
El propósito de este trabajo es describir una experiencia exitosa de la enseñanza y el aprendizaje aplicado a un grupo de estudiantes de secundaria con el uso del software educativo Modellus para simular una reacción redox utilizando ácido cítrico con electrodos de hierro y cobre (batería) y con este objetivo de estudiar el comportamiento del sistema a través de los valores de energía potencial (DDP) que se proporcionan mediante modelado basado en el volumen de solución y compararlos con los valores obtenidos experimentalmente. Se observa entonces que el proceso de creación de modelos de computadora va más allá de una mejora sólo en el conocimiento teórico de los estudiantes, animándoles a la pregunta sobre toda la teoría que participan en la creación del proceso de modelado, ofreciendo sugerencias y buscar soluciones para evitar algunos problemas de y las limitaciones del software. Por lo tanto, los estudiantes se toman más allá de un aprendizaje puramente teórico y abstracto, permitiendo que los estudiantes se apropien de los fundamentos matemáticos y físicos que intervienen en el proceso de enseñanza y aprendizaje, el desarrollo de un ojo crítico y establecer su autonomía a través del diálogo entre sus pares y el maestro.
\end{abstract}

Palabras clave: Software educativo; Aprendizaje; Modelado computacional; Modellus. 


\section{INTRODUÇÃO}

O objetivo deste trabalho é fazer uma simulação do princípio de funcionamento de uma pilha (gerador químico), que utiliza o processo de oxirredução entre o ferro $\left(\mathrm{Fe}^{2+}\right)$ e o cobre $\left(\mathrm{Cu}^{2+}\right)$, em meio ácido, utilizando o software educacional Modellus. Com a simulação pronta, será possível comparar os dados referentes à diferença de potencial (ddp) obtidos através da modelagem computacional e os que foram obtidos experimentalmente a fim de atestar qualitativamentee quantitativamente a eficiência do software e do modelo matemático desenvolvido.

Atualmente, na era tecnológica, observa-se um contexto que engloba inovações tecnológicas aliadas às diversas linhas de pesquisas, cujas finalidades são de aprimorar, desenvolver e incluir os softwares educacionais no ambiente escolar. Neste contexto há iniciativas públicas através do ProInfo, que inicialmente foi chamado de Programa Nacional de Informática na Educação, com a finalidade de disseminar o uso pedagógico das tecnologias de informática e telecomunicações nas escolas públicas de ensino fundamental e médio pertencentes às redes estadual e municipal, (BRASIL. Lei $\mathrm{n}^{\circ}$ 522,1997). A partir de 2007, com a criação de um decreto de Lei(conhecido como Lei do ProInfo), oProInfo passou a ser chamado de Programa Nacional de Tecnologia Educacional, tendo como principal objetivo promover o uso pedagógico das tecnologias de informação e comunicação nas redes públicas de educação básica (BRASIL. Lei nº 6.300, 2007).

Temos conhecimento de políticas públicas que visam a aplicação das Tecnologias da Informação e Comunicação (TIC) nas escolas, porém estas não foram plenamente alcançadas como indica o Relatório de Avaliação da Execução de Programas do Governo $\mathrm{n}^{\circ} 16-$ Infraestrutura de Tecnologia da Informação para Educação Básica (ProInfo)(Controladoria Geral da União 2013), relata que dos 56.510 laboratórios presentes na base de dados fornecidos pela SEED (extinta Secretaria de Educação a Distância) existem em funcionamento27.487, que foram entregues e não instalados 12.610, pelo ao menos 4.332 são utilizados para outras atividades que fogem as aulas de matérias regulares ou de informática e o relatório também aponta a falta de capacitação de professores e técnicos que atingem 15.345 laboratórios. 
De acordo com TíNIO (2003), apesar das dificuldades expostas, as (TIC) quando são empregadas corretamente nas salas de aula tem o potencial de contribuir para o aumento do rendimento dos alunos. Seguindo SIMÕES e GARCÍA (2014) estas tecnologias impelem uma mudança no sistema educacional visando que os professores explorem ao máximo estes recursos tecnológicos nas suas práticas pedagógicas, pois estas, em conjunto com o trabalho dos professores podem vir a despertar o interesse e a motivação dos alunos, principalmente através do acesso irrestrito e imediato as formas de conhecimento via internet. Sendo justamente no seu aspecto comunicativo, através da troca de ideias, dúvidas e sugestões que as modernas Tecnologias de comunicação (TIC) estão ajudando a mudar o cenário do ensino e a aprendizagem. Para ALVES e NOVOS (2003) as possibilidades de acesso à informação e conhecimentos sistemáticos, assim como as interações entre alunos e professores ampliaramse significativamente. A relevância destas atividades também tem sido reconhecida pelo governo brasileiro, como pode-se observar nos Parâmetros Curriculares Nacionais (PCN) para o ensino de ciências. Os PCN de ciências naturais deixam claro que para o ensino da área são procedimentos fundamentais a investigação, curiosidade, interação social, comunicação, o debate de fatos e ideias, respeito à diversidade de opiniões e a busca de provas através da observação, experimentação, comparação, estabelecimento de relações entre fenômenos ou fatos (MEC,2010).

Esta atitude menos passiva em sala de aula é uma clara indicação da relevância pedagógica das atividades práticas. Assim a aplicabilidade do software Modellus, que é uma plataforma de modelagem computacional gratuita, onde a partir de um modelo matemático simples permite-se explorar os dados experimentais gerados através de imagens, animações, gráficos e tabelas ${ }^{1}$. A simulação, portanto, começa a ocupar lugar de destaque nos processos de ensino e aprendizagem, pois a manipulação de parâmetros que lhe é intrínseca, permite experimentar diferentes variáveis para situações do mundo real, oferece comandos que auxiliam no estabelecimento de relações de proporção, tempo, distância e deslocamento, aspectos fundamentais para a compreensão e uso da linguagem das ciências naturais, não se limitando, porém, nem a esta, nem àqueles, mas operando com fronteiras móveis articuladas pelas descobertas a partir da refutação ou da corroboração das hipóteses formuladas e testadas para cada fenômeno apreciado. Recentemente, (Henriques V. B. et.al.2014), mostrou a relevância e a atualidade da proposta de ensino que coloque o 'estudante em ação', com o uso

${ }^{1}$ Para saber mais sobre o Modellus e download consulte na bibliografia (TEODORO, V. D. et al 2016). 
de metodologias que podem ser chamadas de "aprendizagem ativa"2. Acredita-se que a atividade pratica, tanto no laboratório físico através da experimentação quanto no laboratório virtual, através da simulação, constitui uma importante ferramenta para a aprendizagem ativa.

É neste contexto que se apresenta, em seguida, um exemplo concreto de aula envolvendo a metodologia da atividade prática, que utiliza a experimentação com o objeto físico, real, e a simulação com o objeto virtual, proporcionando uma aprendizagem ativa. Uma vez esclarecida a importância e os benefícios do uso dos softwares educacionais para os alunos e professores, pode-se começar a explorar a parte teórica necessária para a criação desta modelagem e realização do experimento.

\section{Princípio de Funcionamento de um Gerador Químico (Pilha)}

A conversão de energia química em elétrica é um processo espontâneo, denominado pilha (célula galvânica). De acordo com FONSECA(2013), só se pode compreender o funcionamento de uma pilha se antes há o conhecimento das reações de oxirredução, que são processos de transferência de elétrons entre átomos e/ou íons das substâncias reagentes. Seguindo FELTRE(2005),parte-se das reações de oxirredução, onde existe um reagente com a tendência de doar elétrons (processo de oxidação) que o transforma no polo negativo da pilha, e outro reagente com a tendência de receber estes elétrons (processo de redução) que é o polo positivo da pilha(Reação I). Estes fenômenos ocorrem simultaneamente, criando assim um fluxo de elétrons. A pilha que foi idealizada neste artigo é constituída por uma lâmina de ferro (dimensões desprezíveis - Figura V) ligada por um fio a uma lâmina de cobre (dimensões desprezíveis- Figura V), ambas imersas em uma solução eletrolítica (suco de limão) responsável por liberar íons $\mathrm{H}^{+}$, permitindo a formação de uma solução condutora de elétrons de uma placa a outra. Com isto, os átomos da placa de ferro passam para o suco de limão perdendo dois elétrons que irão compor a corrente elétrica e o ferro vai para a solução como íons $\mathrm{Fe}^{2+}$. Esses elétrons chegam à placa de cobre onde encontram os íons positivos $\mathrm{H}^{+}($advindos da solução eletrolítica),que estão aderidos à superfície do eletrodo de cobre e os reduzem, dando origem a moléculas de gás hidrogênio $\left(\mathrm{H}_{2(\mathrm{~g})}\right)$.

\footnotetext{
${ }^{2}$ Aprendizagem ativa é um termo técnico para um conjunto de práticas pedagógicas que abordam a questão da aprendizagem pelos alunos sob uma perspectiva diferente das técnicas clássicas de aprendizagem, tais como aulas discursivas, onde espera-se que o professor "ensine" e o aluno "aprenda". Na aprendizagem ativa, entendese que o aluno não deve ser meramente um "recebedor" de informações, mas deve se engajar de maneira ativa na aquisição do conhecimento, focando seus objetivos e indo atrás do conhecimento de maneira proativa.
} 
Reação de oxirredução entre o ferro e o cobre

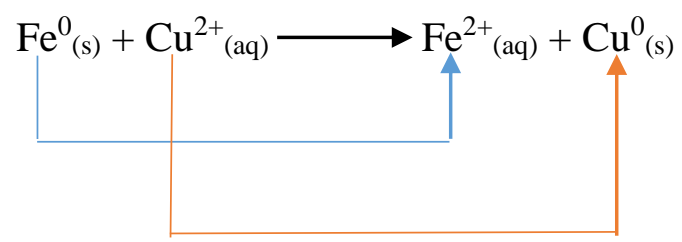

(Reação I)

$\mathrm{Na}$ físico-química pode-se determinar facilmente o valor da diferença potencial de uma pilha através dos valores padrões já tabelados ${ }^{3}$. Segundo ATKINS e LORETTA(2006), esses valores foram medidos relativamente a partir de um padrão, o eletrodo padrão de hidrogênio, escolhido pelo fato do seu cátion $\mathrm{H}^{+}$ser formado a partir da dissociação da água e também por ele ser a espécie mais comum, ao qual foi atribuído o valor arbitrário de 0.00 Volts. A Reação II descreve a formação do cátion $\mathrm{H}^{+}$a partir da dissociação da água $\left(\mathrm{H}_{2} \mathrm{O}\right)$.

Reação de dissociação da água

$$
\mathbf{H}_{2} \mathrm{O} \leftrightarrow \mathrm{H}^{+}+\mathbf{O H}^{-}
$$

(Reação II)

Com esses conceitos podemos construir uma pilha usando um eletrodo inerte de hidrogênio e outro eletrodo de um metal a fim de determinar a sua diferença de potencial. Os elétrons saem da placa do metal e passam pelo voltímetro onde o seu valor é determinado, sendo que o valor medido no voltímetro $(0,44 \mathrm{~V})$ é o potencial padrão $\left(\mathrm{E}^{\circ}\right)$ de oxirredução do ferro. Este mesmo experimento foi realizado com outros metais, dando origem a tabela de potenciais padrão ${ }^{3}$ de oxirredução dos elementos químicos. Por questões de simplicidade, só reproduziremos aqui os valores para oxirredução do ferro (Reação III) e do cobre (Reação IV) que foram medidos para soluções aquosas $1 \mathrm{~mol} / 1,25{ }^{\circ} \mathrm{C}$ e 1 atm, segundo ATKINS e LORETTA(2006):

$$
\begin{aligned}
& \text { Reação de redução do } \mathrm{Fe}^{2+} \text { a Fe } \\
& \mathbf{F e}^{2+}+\mathbf{2 e} \rightarrow \mathbf{F e}^{\mathbf{0}} \quad \mathbf{E}^{\mathbf{o}}=\mathbf{- 0 , 4 4 V}
\end{aligned}
$$

\footnotetext{
${ }^{3}$ Para consultar a tabela de potenciais padrão de redução, veja em ATKINS, P e JONES, L. Princípios de Química: Questionando a Vida Moderna e o Meio Ambiente.Porto Alegre: Editora Bookman, 2006, p 551.
} 
Reação de redução do $\mathrm{Cu}^{2+} \mathrm{a} \mathrm{Cu}^{0}$ :

$$
\mathrm{Cu}^{2+}+2 \mathrm{e} \rightarrow \mathrm{Cu}^{0} \quad \mathrm{E}^{\mathrm{o}}=+\mathbf{0 , 3 4 \mathrm { V }}
$$

Com a finalidade dese determinar a diferença de potencial (ddp) de uma reação de oxirredução entre o ferro e o cobre (Reação I) algumas etapas terão que ser atingidas: primeiro deve-se obter a equação global do sistema, considerando que o ferro por possuir o menor potencial de redução $(-0,44 \mathrm{~V})$ irá liberar os seus dois elétrons, caracterizando o processo de oxidação, logo a Reação III será rescrita com o seu sentido invertido (Reação V). Na etapa seguinte, somamos a Reação V (oxidação do ferro) com a Reação IV (redução do cobre) gerando a equação global da pilha (Reação VI).

\begin{tabular}{|c|c|c|c|}
\hline $\begin{array}{l}\text { Redução do cobre: } \\
\text { Oxidação do ferro: }\end{array}$ & $\begin{array}{l}2 \mathrm{e}+\mathrm{Cu}^{2+} \rightarrow \mathrm{Cu}^{0} \\
\mathrm{Fe}^{0} \rightarrow \mathrm{Fe}^{2+}+2 \mathrm{e}\end{array}$ & $\begin{array}{l}E^{0}=+0,34 V \\
E^{o}=+0,44 V\end{array}$ & $\begin{array}{l}\text { (Reação IV) } \\
\text { (Reação V) }\end{array}$ \\
\hline eação global & $\mathbf{F e}(s)+\mathrm{Cu}_{(\mathbf{a q})}^{2+}$ & $\mathrm{Fe}^{2+}{ }_{(\mathrm{aq})}+\mathrm{Cu}_{(\mathrm{s}}^{0}$ & (Reação VI) \\
\hline
\end{tabular}

Para se determinar a ddp na Reação VI substuímos os valores dos potenciais de redução obtidos na Reação III $(-0,44 \mathrm{~V})$ e Reação IV $(+0,34 \mathrm{~V})$ e aplicamos na Equação I (equação que determina o valor da ddp da Reação Global), onde obtém-se valor de 0,78 V.

$$
\begin{array}{ll}
\Delta \mathrm{E}^{0} & =\mathrm{E}_{\text {ddp de redução maior }}^{0}-\mathrm{E}_{\text {ddp de redução menor. }}^{0} \quad \text { (Equação I) } \\
\Delta \mathrm{E}^{0}=0,34 \mathrm{~V}-(-0,44) \mathrm{V} & \\
\Delta \mathrm{E}^{0}=0,78 \mathrm{~V} &
\end{array}
$$

A modelagem computacional foi construída a partir da equação de Nernst (equação II) que nos possibilita calcular a variação de potencial de uma pilhaa partir das concentrações dos eletrodos em quantidade de matéria. Há de se ressaltar, de acordo com SEEDUC-RJ (2012), a equação de Nernst não é vista pela grande maioria dos alunos do ensino médio no Estado do Rio de Janeiro, não fazendo parte do currículo mínimo de química para o ensino médio e nem está presente nos livros mais recentes que foram usados para embasar este artigo. Em vista disto, apresentaremos a equação de Nernst de uma forma genérica (equação II):

Equação de Nernt:

$$
\Delta \mathbf{E}=\Delta \mathbf{E}^{0}-\frac{R \cdot T}{n_{\cdot} \cdot \mathbf{F}} \cdot \ln \frac{[\mathrm{C}]^{\mathrm{c}} \cdot[\mathrm{D}]^{\mathrm{d}}}{[\mathrm{A}]^{\mathrm{a}} \cdot[\mathrm{B}]^{\mathrm{b}}}
$$


Onde $\mathrm{R}$ é a constante dos gases $(8,31 \mathrm{~J} / \mathrm{K} . \mathrm{mol})$, $\mathrm{T}$ a temperatura em Kelvin, $\mathrm{n}$ é o número de elétrons transferidos entre as espécies envolvidas, F é a constante de Faraday $(96485 \mathrm{C} / \mathrm{mol}),[\mathrm{A}]^{\mathrm{a}}{ }_{,}[\mathrm{B}]^{\mathrm{b}},[\mathrm{C}]^{\mathrm{c}} \mathrm{e}[\mathrm{D}]^{\mathrm{d}}$ são as concentrações das espécies elevadas ao seus expoentes estequiométricos.

Considerando o fator de conversão para passar de logaritmo neperiano para logaritmo na base 10 ser igual a 2,30 e a temperatura ser de $25^{\circ} \mathrm{C}$, inserindo as constantes $\mathrm{R}$ e $\mathrm{F}$ e aplicando na Equação II obtemos a Equação III.

$$
\Delta \mathbf{E}=\Delta \mathbf{E}^{0}-\frac{0,059}{\mathbf{n}} \cdot \log \frac{[\mathrm{C}]^{\mathrm{c}} \cdot[\mathrm{D}]^{\mathrm{d}}}{[\mathrm{A}]^{\mathrm{a}} \cdot[\mathrm{B}]^{\mathrm{b}}}
$$

Considerando, no caso abordado por este artigo, a solução condutora expressaapenas em função da concentração do íon $\mathrm{H}^{+}$, já que este íon é o responsável por fornecer o caráter ácido da solução, rescrevemos a equação III em função apenas da concentração do íon $\mathrm{H}^{+}$.

$$
\Delta \mathbf{E}=\Delta \mathbf{E}^{0}-\frac{0,059}{\mathbf{n}} \cdot \log \left[H^{+}\right]
$$

\section{Circuitos Elétricos}

Um circuito elétrico típico é um conjunto em que a energia é transferida de uma fonte (gerador elétrico), para algum dispositivo, tal como uma lâmpada (resistor) (figura 1).

Figura 1: Circuito Elétrico Simples.

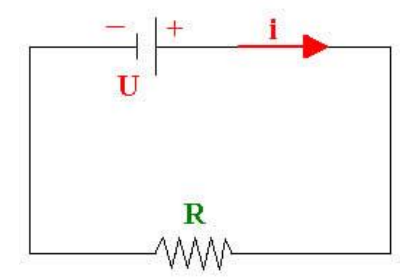

Fonte: SERWAY e JEWETT, 2004.

Como o objetivo é medir o valor da diferença de potencial nos terminais da pilha usase um voltímetro que é ligado em paralelo ao trecho que se deseja medir a tensão elétrica. Para não atrapalhar o circuito, a resistência interna do voltímetro deve ser muito alta 
comparada com a resistência do circuito, para que o aparelho seja considerado ideal (figura 2).

Figura 2: Circuito elétrico com um voltímetro em paralelo.

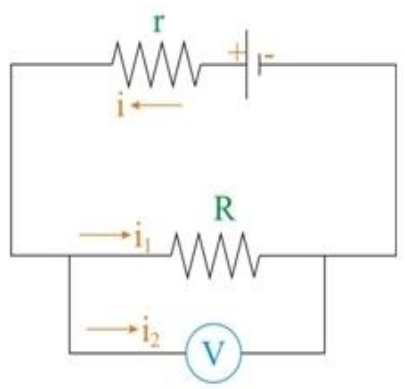

Fonte: RIBEIRO, 2016

Um voltímetro digital é constituído de um galvanômetro associado em série a um resistor, o qual permite medidas de ddp maiores do que a diferença de potencial máxima. $\mathrm{O}$ galvanômetro usado é o de D’Arsonval, que é constituído essencialmente por uma bobina de um fio muito fino, imersa em um campo magnético uniforme de um imã permanente e montado em um sistema de suspensão que o permite girar em torno de um eixo que passa através do seu diâmetro, quando o mesmo é percorrido pela corrente elétrica (figura 3).

Figura 3: Galvanômetro de D’Arsonval.

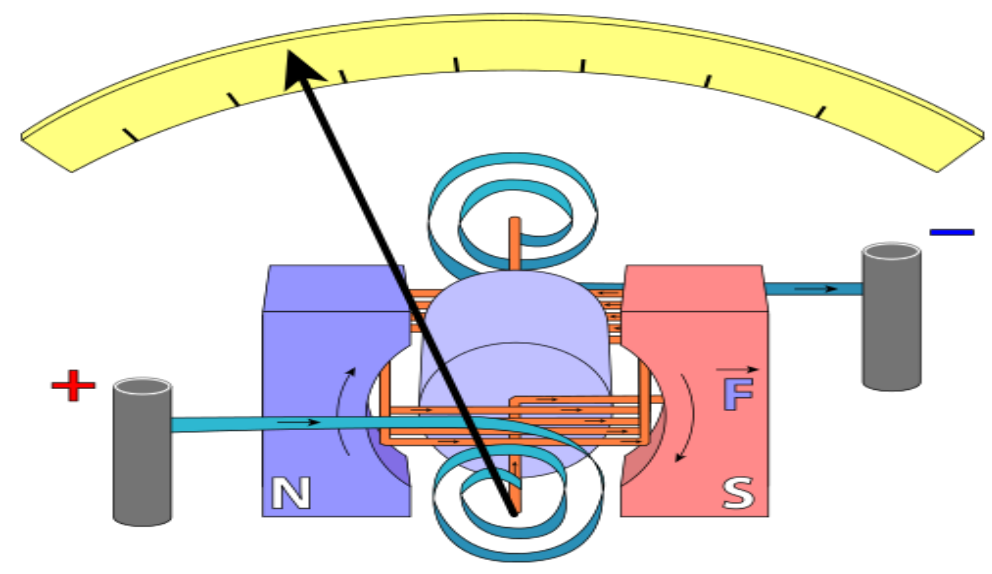

Fonte: SALA DE DEMONSTRAÇÕES DE FÍSICA, 2016.

Esta corrente, que é a corrente que se deseja determinar, produz um campo magnético, o qual interage com o campo magnético do imã permanente, gerando assim um sinal de tensão elétrica que passa por conversor A/D (analógico/digital) que o converte em um sinal 
digital que passará em seguida por um micro controlador que realiza os cálculos matemáticos para medição de cada grandeza elétrica, neste caso a diferença de potencial.

\section{METODOLOGIA}

A metodologia empregada foi subdivida em quatro passo que consistem em criar a modelagem computacional, realizar o experimento, coletar os dados experimentais e a realização de um questionário com os alunos. A ideia de realizar este trabalho foi elaborada após os alunos participantes do Projeto Jovens Talentos da Fundação de Amparo à Pesquisa do Estado do Rio de Janeiro que é desenvolvido em parceria com professores e alunos da Universidade do Estado do Rio de Janeiro, relatarem que nas suas respectivas unidades escolares os professores estavam abordando as reações de oxirredução como tema das suas aulas.

\section{Processo de criação da modelagem computacional- Primeiro passo}

Visto que os alunos adquiriram todo conhecimento teórico necessário através das aulas ministradas nas suas unidades escolares e pelo complemento didático fornecido pelos alunos de licenciatura em Física e Química envolvidos no projeto PIBID-UERJ, pode-se passar para o processo de criação da modelagem computacional. Escolhemos o software educacional Modellus devido ao fato de ser um freeware (software gratuito) que permite a criação de animações, apresentação de figuras e a obtenção de dados experimentais precisos, a partir de um modelo matemático no qual todas as equações, variáveis e constantes necessárias para criação da modelagem devem ser inseridas. $O$ dado experimental gerado nesta simulação (valor da ddp da pilha) é uma resposta em função de uma variável de entrada (volume de suco) que possibilita a alteração de suas quantidades, através de uma barra de controle (Figura V),gerando um determinado valor para a diferença de potencial.

Modelo matemático - No processo de criação, insere-se a variável do sistema, que é o volume de suco (representado pela letra V na figura 4), em seguida as constantes representadas pelos valores da diferença de potencial padrão do ferro e do cobre e o número de elétrons envolvidos na reação de oxirredução (Epf, Epc e n, respectivamente no modelo matemático). Finalmente escreve-se as equações que são necessárias: (as numerações das equações abaixo é a mesma no modelo matemático da Figura 4)

I - cálculo da massa de ácido. 
$m=V[m l] \cdot 1,66\left[\frac{g}{m l}\right] \cdot 0,06$

Onde 1,66 é a densidade do ácido cítrico ${ }^{4}$ e 0,06 é a percentagem de ácido cítrico ${ }^{5}$

II -número de mols do ácido.

$n=\frac{m[g]}{192,124\left[\frac{g}{m o l}\right]}$

III -ddp da reação global.

$\Delta \mathrm{E}^{0}=E p c-E p f$

(Equação 3.3)

Onde Epc é o valor da ddp padrão de redução do cobre. Epf é o valor da ddp padrão de redução do ferro.

IV - concentração do ácido.

O ácido cítrico é um ácido triprótico, logo sofrerá três dissociações seguidas (Reações VII, VIII e IX) até atingir a sua dissociação completa (Reação X), onde observa-se que pela estequiometria que um mol de ácido cítrico está para três mols de hidrônio. $\mathrm{O}$ hidrônio $\left(\mathrm{H}_{3} \mathrm{O}^{+}\right)$ é o responsável por conferir acidez a solução, uma vez que ele se dissocia e libera o íon $\mathrm{H}^{+}$ (Reação XI).

Primeira dissociação do ácido cítrico

Segunda dissociação do ácido cítrico

$$
\begin{aligned}
& \mathrm{C}_{6} \mathrm{H}_{8} \mathrm{O}_{7}+\mathrm{H}_{2} \mathrm{O} \rightarrow \mathrm{C}_{6} \mathrm{H}_{7} \mathrm{O}_{7}^{-}+\mathrm{H}_{3} \mathrm{O}^{+}(\text {Reação VII }) \\
& \mathrm{C}_{6} \mathrm{H}_{7} \mathrm{O}_{7}^{-}+\mathrm{H}_{2} \mathrm{O} \rightarrow \mathrm{C}_{6} \mathrm{H}_{6} \mathrm{O}_{7}^{-}+\mathrm{H}_{3} \mathrm{O}^{+} \text {(Reação VIII) }
\end{aligned}
$$

${ }^{4}$ Densidade do ácido cítrico. Fonte: Aplicações do ácido cítrico na indústria de alimentos. FoodIngredients Brasil. No 30 - 2014. Disponível em <http://www.revista-fi.com/materias/402.pdf>. Acessado em 30 de maio de 2016.

${ }^{5}$ Percentagem do ácido cítrico no limão. Fonte: TRUCOM, C. Ácido Cítrico do Limão. Um agente alcalinizaste,

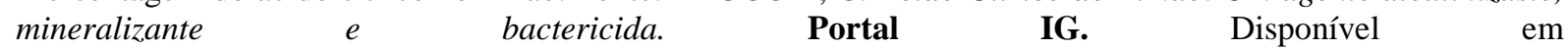
$\langle$ http://somostodosum.ig.com.br/conteudo/c.asp?id=5455>. Acessado em 30 de maio de 2016. 


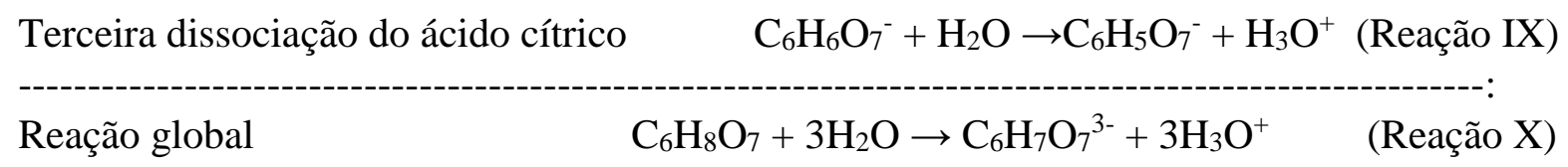

Dissociação do hidrônio:

$3 \mathrm{H}_{3} \mathrm{O}^{+} \rightarrow 3 \mathrm{H}_{2} \mathrm{O}+3 \mathrm{H}^{+}$

(Reação XI)

Concentração do ácido será a mesma que a do íon $\mathrm{H}^{+}$que é dado por:

$$
\left[H^{+}\right]=C A=3 \cdot \frac{n}{V}
$$

V - Equação de Nernst - para o cálculo da diferença de potencial do sistema como um todo, usamos a Equação IV que foi descrita na introdução.

$$
\Delta \mathrm{E}=\Delta \mathrm{E}^{0}-\frac{0,059}{\mathrm{n}} \cdot \log \left[H^{+}\right]
$$

A figura 4 representa o aspecto do modelo matemático final: 
Figura 4: Modelo matemático.

\begin{tabular}{|c|c|}
\hline Modelo Matemático & $=$ \\
\hline $\begin{array}{l}\text { 1-Variaveis } \\
1.1 \text {-Volume de suco } \\
\text { V }\end{array}$ & \\
\hline $\begin{array}{l}\text { 2-Constantes } \\
2.1-D D P \text { do ferro } \\
\text { Epf }=-0.44 \\
2.2-D D P \text { do cobre } \\
\text { Epc }=0.34 \\
2.3-n u m e r o \text { de eletrons envolvidos na reacao } \\
n=2\end{array}$ & \\
\hline $\begin{array}{l}\text { 3-Equacoes } \\
\text { 3.1-Calculo da massa de acido } \\
m=(1.66 \times V) \times(0.06) \\
\text { 3.2-numero de mols do acido } \\
n=\frac{m}{192.124} \\
\text { 3.3-DDP da reacao global } \\
\text { dEp }=\text { Epc - Epf } \\
\text { 3.4-Concentracao do acido } \\
C A=3 \times \frac{n}{V} \\
Q=C A \\
\text { 3.5-Equacao de nernst } \\
E=d E p-\left(\frac{0.059}{n} \times \log (Q)\right)\end{array}$ & \\
\hline
\end{tabular}

Fonte: Própria.

Após o processo de criação do modelo matemático insere-se na interface do programa (barra de controle da figura 5) a variável V (volume de suco) que é o responsável por gerar os valores da diferença de potencial que se deseja observar. As imagens, animações e caixas de texto também são inseridas nesta fase a fim de que se facilite a compreensão do processo analisado. A modelagem assume o seguinte aspecto final: 
Figura 5: Aspecto final da modelagem.

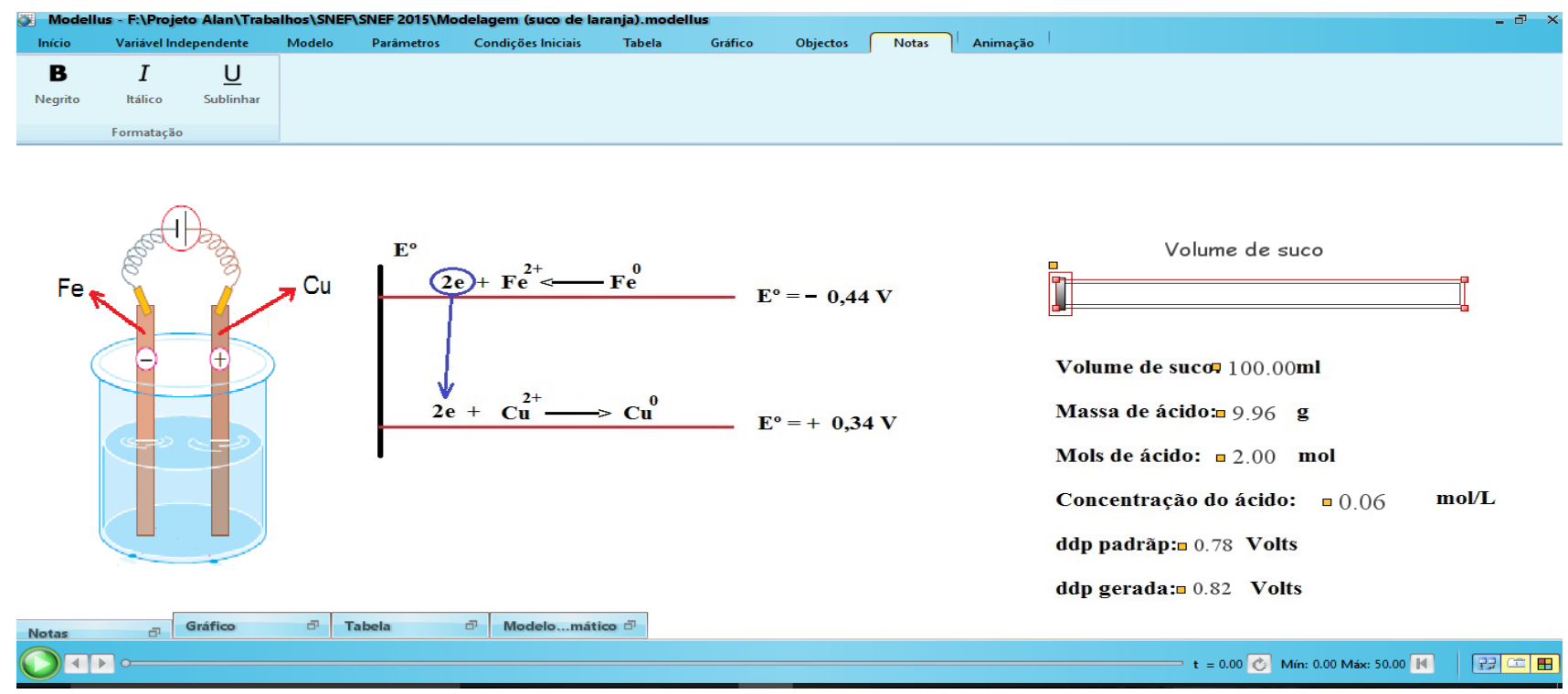

Fonte: Própria.

A única variável que existe na modelagem é o volume de suco, logo se há alterações no seu valor os outros valores mostrados na figura 5 também sofrerão alterações proporcionais à mudança, exceto o valor para ddp padrão que é constante e igual a $0,78 \mathrm{~V}$.

\section{Realização do experimento - segundo passo}

A realização do experimento é um passo determinante para atestar a qualidade dos dados obtidos através da modelagem computacional e também incentivar uma maior participação dos alunos nos processos de ensino e aprendizagem, uma vez que a aula prática muita das vezes consegue atrair mais a atenção dos alunos.

\section{a) Material:}

- 8 limões

- 1 fio de cobre descascado (20,0 cm de comprimento $\mathrm{x} 0,3 \mathrm{~cm}$ de diâmetro)

- 1 fio de ferro descascado (20,0 cm de comprimento $\mathrm{x} 0,2 \mathrm{~cm}$ de diâmetro)

- 2 garras jacarés

- $\quad 2$ béqueres grandes (mais de $500 \mathrm{ml}$ )

- 1 béquer com graduação de 100 mililitros $(100 \mathrm{ml})$ 
- $\quad 1$ voltímetro ${ }^{6}$

b) Procedimento: prende-se o fio de cobre na lateral de um dos béqueres grandes e na lateral diametralmente oposta prende-se o fio de ferro. No outro béquer grande deve ser posto o suco de limão (aqui 8 limões foram suficientes para obter mais de $500 \mathrm{ml}$ de suco), com o béquer pequeno transfere-se $100 \mathrm{ml}$ de suco para o béquer, que já contém os fios. Feito isto, usa-se o voltímetro para medir a ddp do sistema e anota-se. Na etapa seguinte, transfere-se mais $100 \mathrm{ml}$ (totalizando $200 \mathrm{ml}$ ) para o béquer com os fios e mede-se novamente a ddp do sistema. Esse procedimento deve ser repetido mais três vezes até que se totalize $500 \mathrm{ml}$ no béquer com os fios.

\section{Coletas de dados -Terceiro Passo}

Os valores das diferenças de potenciais correspondentes aos volumes de $100 \mathrm{ml}, 200$ $\mathrm{ml}, 300 \mathrm{ml}, 400 \mathrm{ml}$ e $500 \mathrm{ml}$, obtidos tanto pela modelagem quanto pelo experimento foram anotados e relacionados em uma tabela (Tabela I) que será analisada detalhadamente no tópico de resultados e discussões.

\section{Realização do questionário -Quarto Passo}

Por fim foi aplicado um questionário para os20 alunos participantes desta etapa do projeto Jovens Talentos. Os resultados obtidos serão apresentados nos gráficos em forma de Pizza e servirão para embasar os resultados e discussões.

\section{RESULTADOS E DISCUSSÕES}

A fim de atestar a qualidade e a precisão dos valores fornecidos pela modelagem computacional, realizou-se um experimento no qual as variáveis de entrada foram as mesmas da modelagem. A partir disto, foi possível construir uma tabela (Tabela I) comparativa entre os dados obtidos para a diferença de potencial através da modelagem (coluna 1) e do experimento (coluna 2):

${ }^{6}$ Foi utilizado um multímetro digital DT 830B, manual disponível em <http://pt.slideshare.net/danielfxa1/manual-do-multimetro-dt830b $>$. Acessado em 28 de maio de 2016. 
Tabela 1: Comparação entre os valores obtidos para a diferença de potencial.

\begin{tabular}{|c|c|c|}
\hline $\begin{array}{c}\text { VOLUME DO SUCO } \\
\text { DE LIMÃO EM ML }\end{array}$ & $\begin{array}{c}\text { DDP OBTIDA } \\
\text { ATRAVÉS DA } \\
\text { MODELAGEM }\end{array}$ & $\begin{array}{c}\text { DDP OBTIDA } \\
\text { ATRAVÉS DO } \\
\text { EXPERIMENTO }\end{array}$ \\
\hline 100 & $0,83 \mathrm{~V}$ & $0,86 \mathrm{~V}$ \\
\hline 200 & $0,84 \mathrm{~V}$ & $0,86 \mathrm{~V}$ \\
\hline 300 & $0,84 \mathrm{~V}$ & $0,87 \mathrm{~V}$ \\
\hline 400 & $0,85 \mathrm{~V}$ & $0,86 \mathrm{~V}$ \\
\hline 500 & $0,85 \mathrm{~V}$ & $0,86 \mathrm{~V}$ \\
\hline
\end{tabular}

Fonte: Própria.

Observa-se que todos os valores obtidos através da modelagem apresentaram variações em função da concentração que ocorreram devido a simplicidade das equações do modelo matemático, já que a diferença de potencial é uma propriedade intensiva e independe da massa do sistema e também porque o modelo não foi baseado em equações de dissociação de ácidos polipróticos (conteúdo visto apenas no ensino superior) o que deixaria as variações mais sutis. Já os valores obtidos através do experimento, só o referente a $300 \mathrm{ml}$ variou 0,01 $\mathrm{V}$ dos demais, algo que pode ser explicado por uma variação natural do multímetro. Comparando os valores da coluna $1 \mathrm{com}$ os da coluna 2, observa-se que o maior desvio é de $3,5 \%$, referente ao valor de $100 \mathrm{ml}$, um valor que é considerado baixo devido a simplicidade dos métodos empregados tais como a aparelhagem (precisão do voltímetro) e também pelo fato que na modelagem computacional foi utilizada a equação para determinar a concentração do ácido que é vista no ensino médio (Equação 3.4) e não equações para concentração de ácidos polipróticos. Os valores constantes usados como parâmetros referem-se apenas ao ácido cítrico presente em maior quantidade no suco de limão, porém, neste suco há outros ácidos e íons que não foram considerados e que contribuem para aumentar a diferença de 
potencial. A impureza dos materiais empregados também representa outro fator, pois usou-se ligas de ferro e cobre cujas composições percentuais não são especificadas, podendo existir em pequenas concentrações outros elementos químicos que ajudam a compor a corrente elétrica.

Os Gráficos 1, 2, 3, 4 e 5, em forma de pizza, expressam as respostas fornecidas pelos alunos, registradas no questionário.

Gráfico 1: o software Modellus é amigável?

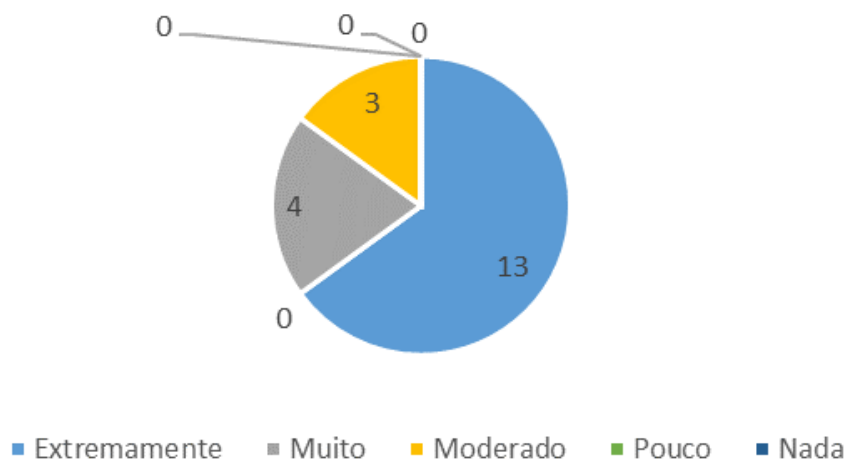

Gráfico 2: como o uso do software foi aceito pelos seus colegas?

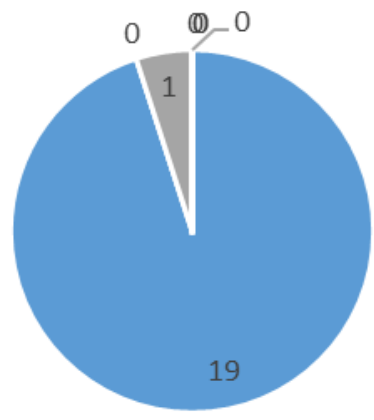

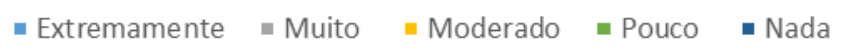


Gráfico 3: quanto o uso do software e a experiência estimularam o seu aprendizado?

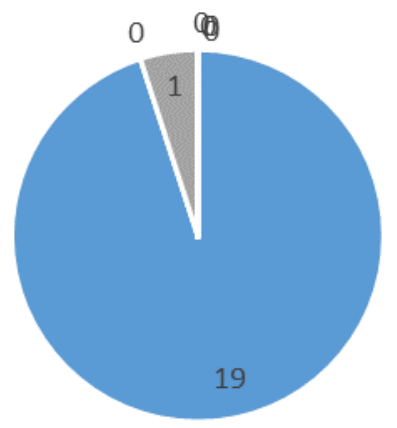

- Extremamente $=$ Muito $=$ Moderado $\because$ Pouco - Nada

Gráfico 4: O uso do software e a experiência ajudaram na compreensão do assunto abordado?

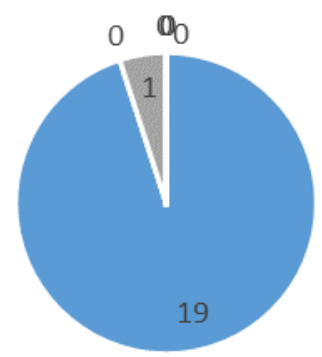

- Extremamente $\mid$ Muito $=$ Moderado $=$ Pouco - Nada

Gráfico 5: As aulas se tornaram mais interessante com o uso do software e da experiência?

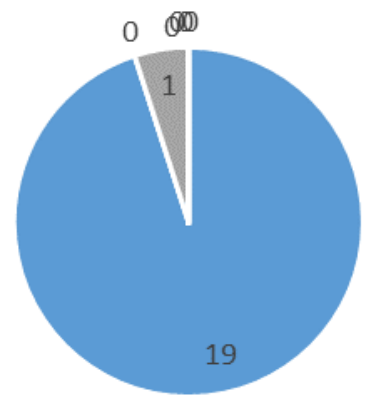

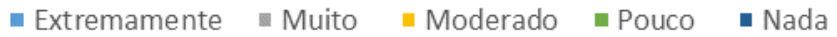


Observando os gráficos notamos que o emprego do computador como ferramenta no processo de ensino e a aprendizagem é bastante estimulante para o aluno no seu processo de ensino e aprendizagem, uma vez que os computadores serviram, segundo retrata VALENTE (1998) como uma ferramenta na qual o aluno desenvolve o conteúdo proposto e, portanto, o aprendizado ocorre pelo fato do mesmo estar executando uma tarefa por intermédio do computador. Fazendo isto através das modelagens computacionais, onde os alunos puderam conhecer melhor as reações químicas, o funcionamento de uma pilha, ao mesmo tempo em que eles foram incentivados a pensar como se daria o seu desenvolvimento, como resolver os problemas para a criação da modelagem, a melhor formar de contornar algumas limitações do software e a pesquisarem mais profundamente os fenômenos físicos e químicos que envolvem o funcionamento desta pilha e o comportamento do voltímetro. Com a realização da experiência, os alunos tiveram a chance de atestar a qualidade da modelagem e também entender o funcionamento do voltímetro, a sua colocação no circuito, observar como alguns parâmetros podem influenciar ou não os valores medidos. Processos de ensino e aprendizagem que favorecem a cognição dos alunos, incentivando-os a procurar próativamente por informações e a desenvolver habilidades e selecioná-las de acordo com a questão que busca responder, aprendendo a aprender (MACHADO et al.,2015).

\section{CONCLUSÃO}

Pode-se então concluir que o uso de softwares para complementar o ensino das ciências exatas, neste caso a física e a química, mostram-se bem eficiente. Os alunos demonstraram uma aprendizagem consistente sobre o tema, pois através do processo de confecção da modelagem eles foram levados a estudar mais sobre as reações de oxirredução, chegando até a equação de Nernst e através do experimento que foi montado puderam compreender o funcionamento de um voltímetro, temas que não são abordados no ensino médio. Durante a elaboração do modelo matemático foi perceptível como o parâmetro variável pode ou não influenciar no sistema, levando-os a buscar na teoria a explicação para os resultados observados. Devido à realização do experimento também foi possível uma comparação entre os dados teóricos e os experimentais, levando os alunos a buscarem novas 
teorias, outros parâmetros e variáveis para explicar a diferença que foi observada entre os valores desses dados. Como os alunos foram estimulados a pesquisar mais sobre as reações de oxirredução, a eletroquímica e o funcionamento do voltímetro através de um estudo mais aprofundado da teoria e das equações usadas, eles puderam fortalecer as correlações entre os diversos fenômenos naturais envolvidos nesta modelagem, favorecendo o dialogismo, beneficiando-os diretamente no processo de ensino e a aprendizagem e no aumento das suas capacidades cognitivas, dando-lhes uma visão mais ampliada do nosso mundo, das tecnologias que nele existem e de como a física e a química estão presentes em nosso cotidiano.

\section{REFERENCIAS}

ALVEZ, L e NOVA, C. Educação a Distância: uma nova concepção de aprendizagem e epistemologia. São Paulo: Editora Futura, 2003.

ATKINS, P e JONES, L. Princípios de Química: Questionando a Vida Moderna e o Meio Ambiente.Porto Alegre: Editora Bookman, 2006, p 549-552.

BRASIL. Lei $\mathrm{n}^{\circ}$ 522, de 9 de abril de 1997. Estabelece a criação do Programa Nacional de Informática na Educação. Diário Oficial [da República Federativa do Brasil], Brasília, DF, No 69, 11 ABR 1997. Seção 1, p 7089 .

BRASIL. Lei $\mathrm{n}^{\circ}$ 6.300, de 12 de dezembro de 2007. Dispões sobre o Programa Nacional de Tecnologia Educacional - ProInfo. Diária Oficial [da República do Brasil], Brasília, DF, № 239, 13 DEZ 2007. Seção 1, p 3.

BRASIL, MISTÉRIO DA EDUCAÇÃO. SECRETARIA DE EDUCAÇÃO FUNDAMENTAL. Parâmetros Curriculares Nacionais: Ciências Naturais. Rio de Janeiro: DP\&A, 2000.

CONTROLADORIA GERAL DA UNIÃO. Relatório de Avaliação da Execução de Programas de Governo no 16 Infraestrutura de Tecnologia para Educação Básica Pública (PROINFO). Brasília, 2013. P 8.

FELTRE, R. Química Volume 2: Físico-Química. 6ª ed. São Paulo: Moderna, 2004. p. 282-305.

FONSECA, M. R. M. Química 2. $1^{a}$ ed. São Paulo: Ática, 2013. p 265.

HENRIQUES, V. B.; PRADO, C. P. C.; VIEIRA, A. P. Editorial Convidativo: aprendizagem ativa. São Paulo: Revista Brasileira de Ensino de Física, 20014, p. 4001-4002.

MACHADO, Alan Freitas; COSTA, Leonardo de moura. A utilização do software MODELLUS no ensino da Física. Interagir: pensando a extensão. Rio de Janeiro. n. 14, 2009. Disponível em: <http://www.epublicacoes.uerj.br/index.php/interagir/article/viewFile/1814/1383>. Acesso em 28 de maio de 2016.

RIBEIRO, T. Voltímetro e Amperímetro. InfoEscola Navegando e Aprendendo.s.d. Disponível em $<$ http://www.infoescola.com/eletricidade/voltimetro-e-amperimetro/>. Acessado em 27 de maio de 2016. 
RIO DE JANEIRO. Currículo Mínimo 2012 Química. Secretaria de Estado de Educação do Rio de Janeiro 2012. Disponível em 〈http://www.rj.gov.br/web/seeduc/exibeconteudo?article-id=759820>. Acesso em: $27 \mathrm{de}$ maio de 2016.

SALA DE DEMONSTRAÇÕES DE FÍSICA. Galvanômetro de D’Arsonval. Departamento de Física da UFMG. I. Disponível em <http://demonstracoes.fisica.ufmg.br/demo/273/5H50.10-Galvanometro-de-DArsonval>. Acessado em 27 de maio de 2016.

SERWAY, R. A.; JEWETTJR, J. W. Princípios de Física: Eletromagnetismo (Vol. 3). São Paulo: Thomsom Learning, 2004. 348 p.

SIMÕeS, D e GARCÍA F. A Pesquisa Científica com Linguagem e Práxis.Rio de Janeiro, Publicações Dialogarts, 2014, p 47 - 62.

TEODORO, V. D.; VIEIRA, J. P. D.; VIEIRA, P. D. Modellus v4.5. Lisboa: Modellus, 2015. Software freeware disponível em: < http://modellus.co/index.php/pt/>. Acesso em 27 de maio de 2016.

TINIO, V. L. ICT in Education. Nova Iorque: Information and Communication Technologies (ICT) for Development, 2003. Disponível em: 〈http://www.saigontre.com/FDFiles/ICT_in_Education.PDF>. Acesso em: 26 de maio de 2016.

VALENTE, J. A. Computadores e Conhecimento: representando a educação. São Paulo: Gráfica Central UNICAMP, 1998. 